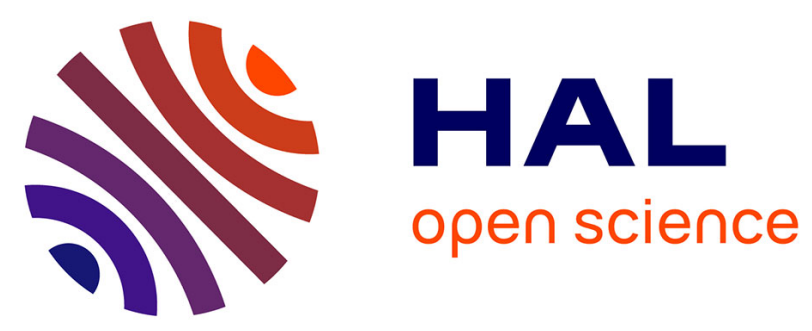

\title{
Norm-Based Approach to Incorporate Human Factors into Clinical Pathway: Reducing Human Error and Improving Patient Safety
}

Jasmine Tehrani, Vaughan Michell, Yu-Chun Pan

\section{To cite this version:}

Jasmine Tehrani, Vaughan Michell, Yu-Chun Pan. Norm-Based Approach to Incorporate Human Factors into Clinical Pathway: Reducing Human Error and Improving Patient Safety. 18th International Conference on Informatics and Semiotics in Organisations (ICISO), Jul 2018, Reading, United Kingdom. pp.73-82, 10.1007/978-3-319-94541-5_8 . hal-01920714

\section{HAL Id: hal-01920714 \\ https://hal.inria.fr/hal-01920714}

Submitted on 13 Nov 2018

HAL is a multi-disciplinary open access archive for the deposit and dissemination of scientific research documents, whether they are published or not. The documents may come from teaching and research institutions in France or abroad, or from public or private research centers.
L'archive ouverte pluridisciplinaire HAL, est destinée au dépôt et à la diffusion de documents scientifiques de niveau recherche, publiés ou non, émanant des établissements d'enseignement et de recherche français ou étrangers, des laboratoires publics ou privés. 


\title{
Norm-based Approach to Incorporate Human Factors into Clinical Pathway: Reducing Human Error and Improving Patient Safety
}

\author{
Jasmine Tehrani ${ }^{1}$, Vaughan Michell ${ }^{2}$ and Yu-Chun Pan $^{3}$ \\ ${ }^{1}$ University of Bedfordshire, Luton, LU1 3JU, UK \\ ${ }^{2}$ University of Reading, Reading, RG6 6UR, UK \\ ${ }^{3}$ University of west London, London, W5 5RF, UK \\ Jasmine.hajrezatehrani@beds.ac.uk \\ v.a.michell@henley.ac.uk \\ yu-chun.pan@uwl.ac.uk
}

\begin{abstract}
Patient safety and accidental harm or iatrogenic errors are increasingly important healthcare issues resulting in high costs and mortality. The way clinical workflow and actions are communicated can impact patient safety. Although much work has been done to identify the individual human factors and recommendations are made to control and reduce human factor errors, little work has been done to provide a structured methodology to analyse and control human factor influencing patient safety outcomes. In this paper, we build on the previous work on automatic development of clinical pathways, semiotic approach to modelling norm-base clinical pathways and propose a Human Factor Failure Modes and Effects Analysis (HFMA) which offers a systematic approach to define, design and incorporation of human factors into formal design of clinical pathways. Organisational semiotics methods specifically NAM and SAM are applied to identify and analyse controls to reduce the adverse impact of human factors in healthcare settings. This is achieved through modelling and integration of human factors into clinical pathways. This will result in more rigorous control the care process ensuring completeness, consistency and patient safety by enabling the mapping of formal and informal/safety controls into clinical pathways.
\end{abstract}

Keywords: Clinical Pathways, Process Modelling, Organizational Semiotics, Norm Analysis, Information System, Human Factors.

\section{$1 \quad$ Introduction}

The challenge of achieving significant improvements in patient safety is one of the key tasks facing healthcare at the start of the 21st century. There is broad international agreement on the nature of the task faced and the importance of achieving improvements to quality in this area [1]. Large numbers of people continue to be successfully cared for and treated in the National Health Service, but a significant number of errors and other forms of harm occur. It is calculated that around $10 \%$ of patients admitted to NHS hospitals are subject to a patient safety incident and that up to half of these incidents could have been prevented [2]. Medical errors are also a serious and challenging 
issue in the United States. According to the Institute of Medicine 's (IOM's) recent report, To Err Is Human: Building a Safer Health System (1999), between 44,000 and 98,000 people die in hospitals each year as the result of medical errors. Human factors in the provision of health is responsible for a major part of safety problems since the care activity is handled by practitioners and their ability to process multiple pieces of contradictory information is limited. Therefore, safety in medicine is a rapidly developing field and several interdisciplinary research groups have investigated the effect of human and organisational factors on the reliability of healthcare delivery. The latest survey of published work on human factors disclosed that the estimated contribution of human error to accidents in hazardous technologies increased fourfold from the 1969 [4]. The human factors community has developed a variety of methods which are beginning to be adopted in healthcare setting [3]. One of the main tools developed to manage the care quality in healthcare setting are Clinical pathways (CP), also known as care pathways. It is proven that their implementation reduces the variability in clinical practice and improves outcomes. Despite the substantial improvements in modelling and generation of CPs, there is very little account for human factors [15].

This paper builds on previous work on clinical pathway modelling by presenting a normative approach to the analysis and integration of human factors in to clinical pathways in order to accommodate exceptions which have not been dealt with by other conventional methods [24]. The proposed methodology provides a robust mechanism to analyse human factor failure points and to identify and model the controls in to formal process models e.g. CPs. Norm Analysis Method (NAM) is adopted to analyse patterns of behavior and decision-making models of clinicians and the condition under which the behavior will occur. This mechanism is crucial for conceptualizing and developing personalized clinical pathways which describes the conditions and temporality of human factor failure modes.

\section{Norm Based Approach for Incorporating Human Factors into Clinical Pathways}

In this research, a semiotics method, namely, norm analysis method is chosen to compliment BPMN to enable the modelling of behavior. NAM is used to capture rules, regulations and condition under which every action of an agent is legal, acceptable or prohibited. The norms define a culture or subculture. In a system of agnets, norms reflect regularities in the behaviour of members allowing co-ordination of their actions. Norms are developed through practical involvements of agents in a society and have purposes of directing, coordinating and controlling movements within society [17]. It is proposed that extension of BPMN with norms enables the modelling of modelling a complex business processes. Every activity is made up of one or more norms. Hence it might be difficult to view the relationships between the norms, and how they interact with each other. To improve the practical ability of norms, this research extends the description of process models with norms as a reasonable mechanism to enable the modelling of business dynamics. 
Table 1. Example of norms in a clinical pathway.

\begin{tabular}{|c|c|}
\hline Norm No. & Definition \\
\hline Norm N1 & $\begin{array}{l}\text { Whenever <the patient is assessed for venous thromboembo- } \\
\text { lism> } \\
\text { If <there is bleeding risk > } \\
\text { Then < doctors> is <permitted > to <prescribe prophylaxis> }\end{array}$ \\
\hline Norm N2 & $\begin{array}{l}\text { Whenever < performing pressure ulcer risk assessment }> \\
\text { If <Pressure Ulcer is classified as extensive destruction > } \\
\text { Then <nurse> is <obliged > to <place patient on a Bi- Wave } \\
\text { mattress> }\end{array}$ \\
\hline Norm N3 & $\begin{array}{l}\text { Whenever <performing pressure ulcer risk assessment> } \\
\quad \text { If < patient develops a pressure ulcer during this hospital } \\
\text { stay> } \\
\text { Then < }\end{array}$ \\
\hline
\end{tabular}

Norms define business rules that are imposed on the particular process [23]. For example, in Table 1, Norm N1 reflects rules that must be followed during VTE assessment. Norm N2 includes both the business rules and an exception that will be triggered (caused) when pressure ulcer assessment action has been invoked. Besides handling the business rules and exceptions, the norm provides a degree of flexibility that allows the analysts to model exceptional and alternative situations where decisions are made solely based on human judgment. For example, when performing pressure ulcer risk assessment, if patient develops ulcer during his hospital stay, the nurse is advised to arrange clinical photography. However, the final decision making is solely based on the human agent's judgment. The extension is carried out by incorporating norms into the business process diagram. In the diagram, each control condition is labelled as [N\#] where \# is the number for identification. The labels are then elaborated in the norm specifications to indicate the condition, the actor and action to be undertaken.

\section{$3 \quad$ Human Failure}

Human rather than technical failures now represent the greatest threat to healthcare settings. Managing the human risks will never be $100 \%$ effective. Human fallibility can be moderated, but it cannot be eliminated. It is inevitable that errors will occur in healthcare, as they do in other safety critical industries, because they are an intrinsic human trait [19]. An acceptance of this position towards safety, can lead to the achievement of significant improvements in improving safety measures [28]. In recent years, the focus within adverse event analysis, situations in which error and other forms of harm occur, in safety critical industries have moved from a propensity for individual blame to a systems approach. In fact, accepting the fact that people are liable to make 
errors, system and equipment design, training and other aspects of the work environment are given priority in terms of initiating change to minimise the risk. Furthermore, achieving improvement in patient safety is not possible unless human factors are placed at the heart of improving clinical, managerial and organisational practice leading to improvements in patient safety. Of particular attention is the inner model of clinician's thinking and decision-making models adopted in challenging health provision circumstances, which centres around a complex series of interactions and team-based activities between practitioners and patients as well as numerous technological instruments and information systems that aid decision making and streamline care delivery process. There have been several attempts to include human factors in the analysis of medical errors and patient safety issues. James Reason (1995) analysed conditions under which human factors can contribute safety failures and proposed a generic model of accident causation [19]. Chang et al (2005) conducted a series of similar studies and presented an evaluation of existing patient safety terminologies and classifications and grouped the findings into five complementary root nodes: impact, type, domain, cause and prevention [8]. Although various integrated models of error have been produced, few if any focus on the detailed categorisation of the wide range of specific human factors that contribute to error. However, the SHEEP model, was developed from analysis of human factor course participants to identify human factor categories and types that have a bearing on clinical actions [29].

\section{$4 \quad$ Human Factors}

Human factors encompass all factors that can influence people and their behavior. One simple definition is design for human use [9]. Chapanis defines human factors as a body of information about human abilities, limitations and characteristics that are relevant to the design process. In a work context, human factors are the environmental, organisational and job factors, and individual characteristics that influence behavior at work. Human factors in the provision of health is responsible for a major part of safety problems since the care activity is handled by practitioners and their ability to process multiple pieces of contradictory information is limited. Therefore, safety in medicine is a rapidly developing field and several interdisciplinary research groups have investigated the effect of human and organisational factors on the reliability of healthcare delivery [9].

\section{$5 \quad$ Failure Mode and Effects Analysis}

We have seen how human behaviour and human factors contribute to errors and their risk. Errors come together at the confluence of the organisation, workplace and person in the process. They are typically combatted by identifying the risks of process failure in terms of both the planned process and how the execution may differ in practice. The Failure Mode and Effects Analysis method of identifying failures in products and processes has been widely used both in industry and in medicine [8]. In summary it involves the identification of the ways in which a process can fail and the apportioning 
of the risk via the probability a failure of this kind (P), the impact of the failure (I) and the possibility of detection (D). Each of these metrics is typically measured on a scale of 1-10 and then multiplied to provide an overall risk number as high as 1000 as a ranking measure of the risk [29]. Typically, any factor $>7$ is considered a high risk factor contributor. FMEA assumes a process model or documentation is available and that events leading to failure can be identified and that remedial risk reduction actions are also identifiable [20].

\section{Human Failure Modes and Effects Analysis (HFMEA)}

Most FMEA analysis covers human and technology error, but rarely classifies human factors. In this paper, an extension of FMEA is proposed for clinical risks management by identifying a set of human factor potential failure modes hence the proposed methodology is called Human Factors Modes and Effects Analysis (HFMEA). Due to the nature of human error, there are innumerable ways in which human factors can impact a process making it extremely difficult to identify all the potential human factors driven failures and risk protection measures. However, using HFMEA three initial levels of risk are identifiable:

- High: 7+ the patient may die, requiring emergency intervention

- Medium: score 4-6 the patient is impacted resulting in additional significant intervention

- Low 1-3: the patient is inconvenienced resulting in minor changes in intervention to arrive at the original intervention goals to reduce the potential human factors failures to a manageable set to analyse we consider only the high-risk patient related activities.
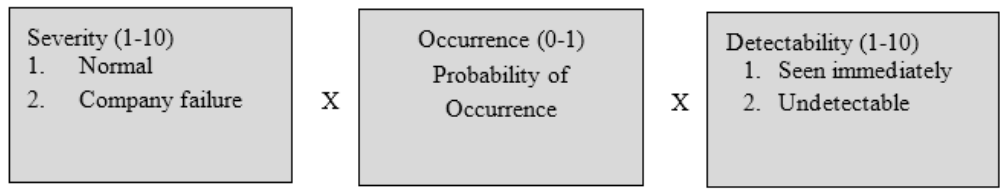

Fig. 1. Failure Mode and Effects Analysis

A high-risk patient activity is considered as an activity where one mistake could lead to a massive adverse impact ( $>7$ ) on the patient if a key action was mistaken. i.e. ' 1 step to disaster'. For example, miscalculating the quantity of a lethal drug to give an overdose, removing the wrong organ. Patient high risk activities also have low detection rates or few steps in which they are detected as a result of few or poor control norms i.e. $\mathrm{D}>7$. But how is $\mathrm{P}>7$ identified. For example, anesthetists and nurses routinely administer lethal drugs with very few failures and hence $\mathrm{P} \ll 1$ or 2 . This is often because the routine nature of the task is just that and there is no significant change in routine leading to unexpected actions or a reduction in perception, evaluative or cognitive capability. As Reason asserts it is often events and unfamiliar or unprepared for 
situations that give rise to safety errors [20]. The key to how $\mathrm{P}$, the probability of a patient safety risk occurring, might suddenly increase is to identify the conditions under which P will drastically increase due to the removal of 'normal' safety measures as a result of a change in the situation or human behavior. This requires identification of the human factor failure points and the controls employed in the formal process - typically a codified clinical pathway. If we can define these high-risk points for each elective clinical process, we can then define the human factor behaviors that could lead to the catastrophic result and design countermeasures to them. An excellent example is the risk of cabin pressure due to external cabin doors not being correctly locked resulting in the now universal safety control countermeasure of ensuring each cabin crew member that checks a door check their opposite number's doors are safely locked or unlocked.

\subsection{Controls}

Errors can be reduced via the use of controls at the individual, process and organisational level. Sadiq et. Al (2000), suggest the need to ensure a systematic approach to business objectives and control objectives in process design. Although Sadiq's focus was compliance controls, it applies equally well to error controls. Our research suggests there is rarely a systemic approach in clinical pathway design and specifically less to error control design. What is needed is explicit analysis and modelling of the process and defined and reasoned control objectives against a defined clinical risk and set of internal controls to reduce the risk. What often happens is that for clinical processes controls in the form of checklists etc. are often added after errors occur and a root cause analysis occurs as a system of reminders to prevent [31].

\subsection{Predictive Controls}

The use of FMEA or other methods can produce a set of 'predictive error control points' where predictive controls are defined as controls able to be set up for a known process ahead of time to catch predicted failures. Many procedures are designed around predictive controls with checkpoints. Predictive controls require a good knowledge of the actual activities and behaviors and their variations, actions and states of the stakeholders and known failure modes. This enables the identification of control objectives and needed controls at appropriate risk points. Predictive controls can be modelled as control norms in the human to human interactions and human to information system interactions and hence can only really be applied well to elective surgery in reliable conditions [24].

The use of FMEA or other methods can produce a set of 'predictive error control points' where predictive controls are defined as controls able to be set up for a known process ahead of time to catch predicted failures. Many procedures are designed around predictive controls with checkpoints. Predictive controls require a good knowledge of the actual activities and behaviors and their variations, actions and states of the stakeholders and known failure modes. This enables the identification of control objectives 
and needed controls at appropriate risk points. Some of the predictive controls are: Process controls: decision points and loops, human monitoring points, Machine monitoring points and memory cues, action sequence rule/knowledge cues. Predictive controls can be modelled as control norms in the human to human interactions and human to information system interactions and hence can only really be applied well to elective surgery in reliable conditions.

\subsection{Personal Controls}

We define a second set of controls as personal controls. We define personal controls as informal control rules or heuristics - i.e. behavioral norms used by the individual to ensure the correct outcome of actions. Personal controls are informal as they are tacit and not formally codified by the organisation. Personal controls depend on the individual's character and self-discipline for their introduction and are typically the result of experience and concern about the outcome of an activity. They are part of the individual's set of behavioral norms [23]. For example, one interviewee on a patient safety survey always verbally repeated drug volume and strength information and asked for a second check whenever they knew themselves to be tired and hence the possibility of a perception or epistemic error was reduced. However, the enactment of her 'personal patient safety control norm' depends on her discipline and awareness, or 'strength of character', also subject to human factor failings such as stress. Hence the need for personal controls .at stress points or points of high risk needs to be highlighted within clinical pathways.

\subsection{Culturally Driven Controls}

A third set of controls is culturally driven controls resulting from national, organisational, professional, or team driven learnt and repeated behaviors. These relate to cultural norms. As Reason asserts organisational methods, actions and traditional working practices can create a communal safety culture. The existence of cultural norm beliefs and accepted behaviours creates discomfort and dissonance if the cultural behaviour or belief is violated. However, it can be difficult to measure or define a safety culture and the 'norms' of safety behaviour. Repeated training, examples and practice and consistent control behaviour can develop cultural controls. Cultural controls can be developed through human factors training and repeated application of good practices and reflection.

\section{$7 \quad$ Risk Alleviation Norms to Improve Patient Safety Outcomes}

As discussed earlier in this chapter, generally clinical pathways refer to medical guidelines. However, a single pathway may refer to guidelines on several topics in a well specified context. CP is a management tool based on evidence-based practice for a precise group of patients with a foreseeable clinical course, in which the different tasks by the professionals involved in the patient care process are defined and sequenced either 
by hour (ED), day (acute care) or visit (homecare). Outcomes are tied to specific interventions. Clinical pathways (integrated care pathways) can be seen as an application of process management thinking to the improvement of patient healthcare. An aim is to recentre the focus on the patient's overall journey, rather than the contribution of each specialty or caring function independently. Instead, all are emphasised to be working together, in the same way as a cross-functional team.

In the healthcare domain, the clinical pathways can be seen as the norms developed through the practical medical experiences of healthcare professionals, since they have functions of directing, coordinating and controlling actions in the healthcare process. These norms will provide guidance for medical care staff staffs to determine whether certain medical behaviours at any given time. For clinical pathway design, these norms are analysed, captured and integrated in to the formal organisational process e.g. clinical pathways serving as a basis for designing norm-base clinical pathways that improves patient safety outcomes by addressing formal/informal human factors that influence patient safety outcomes. We use the notion of norms to analyse and formally structure informal/human factors that influence patient safety outcomes. These norms are called risk alleviation norms, which then integrated into clinical pathways design using the normative approach to incorporate human factors in clinical pathways. In the following sections, the methodology for analysing and structuring of human factors affecting patient safety outcome is described in detail. The HFMEA method, described in previous section, is used as an essential part of this mythology to enable the identification of high risk points within the pathway. Subsequently, after identification of high risk points for each elective clinical process, we can then define the human factors behaviours that could lead to the catastrophic result and design countermeasures to them.

\section{Extension of Clinical Pathways with Risk Alleviating Norms}

Norm analysis method is applied to the analysis and formalisation of human factors. Human factors or factors of human behaviour are a key adverse influence on how clinicians behave, think, make judgements and perceive the world. Although much work has been done to identify the individual human factors and recommendations regarding behaviours to control and reduce human factors errors, little work has been done to provide a structured approach to analyse and develop the human factor behaviour. Hence in this research, a normative approach to regulate and control the effects of human factors on patient safety by modelling and embedding them within clinical pathways as the formal work process models is proposed. The stages involved in this methodology are described in figure below. As it can be seen in figure 1, using HFMEA the high-risk points with the formal process map are identified and each high risk point is further organised into high, medium and low risk patient activity. For high risk points within the process map, risk alleviating norms are used to and integrated into the formal process map to reduce the impact of failure on patient. Using risk alleviating norms, controls are introduced and formally integrated into the clinical pathway design. 


\section{Conclusion}

This paper presented a background to the current application of clinical pathways in hospitals and presented a case for the need to consider human factors if significant improvement in patient safety outcomes are intended. It is argued that integration of human factors in clinical pathways design will have a significant role in improving patient safety. In this research, we have built on the previous work on modelling the dynamic behavior of business organisations by presenting a methodology for extending business process modelling notation with norms to enable the modelling the dynamics of business processes and to accommodate exceptions which have not been dealt with by other conventional methods [23][24]. Norm analysis is adopted as the method for modelling the dynamics of patterns of behaviour which are defined as shared a set of 'norms' which govern how members of the society behave, think, and make judgment [26]. The proposed methodology addresses social and informal/safety factors, which conspire together to influence the outcome of patient safety. To this end, a semiotics-oriented method that adopts organisational semiotics methods, in particular, SAM and NAM are proposed. Semantic analysis method is applied to explicitly represent the semantics of the concepts, their relationships and patterns of behaviour, which offers a basis for analysing human/Informal factors in healthcare setting. Moreover, an extension of FMEA approach is proposed that enables incorporation of human factors failure modes and effects analysis into formal description of clinical pathways (HFMEA). This is the main contribution of this paper which provides a comprehensive platform for analysis and formal strutting of human factors in forms of failure modes and their incorporation into clinical pathways.

\section{References}

1. Milligan, Frank J. "Establishing a Culture for Patient Safety-The Role of Education." Nurse Education Today 27.2 (2007): 95-102.

2. Shaw, R., Et Al. "Adverse Events and Near Miss Reporting In the Nhs." Bmj Quality \& Safety 14.4 (2005): 279-283.

3. West, M. A., Guthrie, J. P., Dawson, J. F., Borrill, C. S. \& Carter, M. 2006. Reducing Patient Mortality in Hospitals: The Role of Human Resource Management. Journal Of Organizational Behavior, 27, 983-1002

4. Hollnagel, E. 1993. Reliability of Cognition: Foundations of Human Reliability Analysis. Academic, London.

5. Abidi, S. S. R. \& Chen, H. 2006. Adaptable Personalized Care Planning Via a Semantic Web Framework. 20th Intlreferences

6. Cabitza, F., Simone, C. \& Sarini, M. 2008. Knowledge Artifacts as Bridges between Theory and Practice: The Clinical Pathway Case. Knowledge Management in Action, 37-50.

7. Carthey, J., Clarke, Julia Field, Campaign; Associate (Safer Care Priority Programme), Nhs Institute For Innovation And, Improvement 2010. Leadership for Safety: Implementing Human Factors In Healthcare.

8. Chang, A., Schyve, P. M., Croteau, R. J., O’leary, D. S. \& Loeb, J. M. 2005. The Jcaho Patient Safety Event Taxonomy: A Standardized Terminology And Classification Schema 
For Near Misses And Adverse Events. International Journal For Quality In Health Care, 17, 95-105.

9. Chapanis, A. (Ed.) 1996. Human Factors In Systems Engineering: Wiley Series In Systems Engineering.

10. Forster, P. 2003. To Err Is Human. Annals Of Human Genetics, 67, 2-4.

11. Helmreich, R. L. 2000. On Error Management: Lessons From Aviation. Bmj: British Medical Journal, 320, 781.

12. Hollnagel, E. 1993. Reliability Of Cognition: Foundations Of Human Reliability Analysis. Academic, London.

13. Hurley, K. F. \& Abidi, S. S. R. Year. Ontology Engineering To Model Clinical Pathways: Towards The Computerization And Execution Of Clinical Pathways. In, 2007. Ieee, 536541.

14. Liu, K. 2000. Semiotics In Information Systems Engineering, Cambridge ; New York, Cambridge University Press.

15. Liu, K. \& Dix, A. Year. Norm Governed Agents In Cscw. In, 1997. Citeseer.

16. Liu, K., Sun, L., Barjis, J. \& Dietz, J. L. G. 2003. Modelling Dynamic Behaviour Of Business Organisations-Extension Of Demo From A Semiotic Perspective. Knowledge-Based Systems, 16, 101-111.

17. Milligan, F. J. 2007. Establishing A Culture For Patient Safety-The Role Of Education. Nurse Education Today, 27, 95-102.

18. Mor Plege, P. C., Anand Kumar 2012. Comparing Computer-Interpretable Guideline Models: A Case Study Approach.

19. Osborn, S. \& Williams, S. Seven Steps To Patient Safety. An Overview Guide For Nhs Staff. Londres: The National Patient Safety Agency 2a Edicion, Abril 2004. Consultado 08-122008). Disponible En: Http://Www. Npsa. Nhs. Uk/Nrls/Improvingpatientsafety/PatientSafety-Tools-And-Guidance/7steps/[Links].

20. Reason, J. 1995. Understanding Adverse Events: Human Factors. Quality In Health Care, 4, 80-89.

21. Sonnenberg, F. \& Hagerty, C. 2006. Computer-Interpretable Clinical Practice Guidelines. Where Are We And Where Are We Going, 145-158.

22. Stamper, R. Year. Social Norms In Requirements Analysis: An Outline Of Measur. In, 1994. Academic Press Professional, Inc., 107-139.

23. Stamper, R. 2009. The Chemistry Of Society: Organisational Semiotics As An Empirical Social Science. 11th International Conference In Informatics And Semiotics In Organisations, An Ifip Wg8.1 Working Conference. Beijing, China.

24. Tehrani J, L. K., Michel V. 2012. Semiotics-Oriented Method For Generation Of Clinical Pathways. International Conference On Logistics, Informatics And Service Sciences (Liss). Beijing, China.

25. Vincent, C., Neale, G. \& Woloshynowych, M. 2001. Adverse Events In British Hospitals: Preliminary Retrospective Record Review. Bmj, 322, 517-519.

26. West, M. A., Guthrie, J. P., Dawson, J. F., Borrill, C. S. \& Carter, M. 2006. Reducing Patient Mortality In Hospitals: The Role Of Human Resource Management. Journal Of Organizational Behavior, 27, 983-1002.

27. Wright, G. H. 1963. Norm And Action: A Logical Enquiry, Humanities Press.

28. Vincent, Charles, Susan Burnett, And Jane Carthey. "Safety Measurement And Monitoring In Healthcare: A Framework To Guide Clinical Teams And Healthcare Organisations In Maintaining Safety." Bmj Qual Saf 23.8 (2014): 670-677.

29. Michell, Vaughan, Ed. Handbook Of Research On Patient Safety And Quality Care Through Health Informatics. Igi Global, 2013. 
